\title{
Abundances of Ultra-Heavy Galactic Cosmic Rays from the SuperTIGER Instrument
}

\author{
R. P. Murphy ${ }^{* 1}$, W. R. Binns ${ }^{1}$, R. G. Bose ${ }^{1}$, T. J. Brandt ${ }^{2}$, P. F. Dowkontt ${ }^{1}$, T. Hams ${ }^{2,6}$, \\ M. H. Israel ${ }^{1}$, A. W. Labrador ${ }^{3}$, J. T. Link ${ }^{2,6}$, R. A. Mewaldt ${ }^{3}$, J. W. Mitchell ${ }^{2}$, B. F.

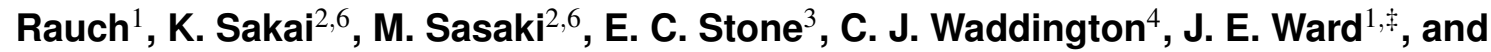 \\ M. E. Wiedenbeck ${ }^{\dagger \dagger}$ \\ ${ }^{1}$ Washington University in St. Louis, St. Louis MO 63130 USA \\ ${ }^{2}$ NASA Goddard Space Flight Center, Greenbelt, MD 20771 USA \\ ${ }^{3}$ California Institute of Technology, Pasadena, CA 91125 USA \\ ${ }^{4}$ University of Minnesota, Minneapolis, MN 55455 USA \\ 5 Jet Propulsion Laboratory, California Institute of Technology, Pasadena, CA 91109 USA \\ ${ }^{6}$ Center for Research and Exploration in Space Science and Technology (CRESST) \\ $¥$ Now at Institut de Fisica d'Altes Energies (IFAE), Bellaterra, Spain
}

E-mail: rmurphỵphysics.wustl .edu

\begin{abstract}
The SuperTIGER (Trans-Iron Galactic Element Recorder) experiment was launched on a longduration balloon flight from Williams Field, Antarctica, on December 8, 2012. SuperTIGER flew for a total of 55 days at a mean atmospheric depth of $4.4 \mathrm{~g} / \mathrm{cm}^{2}$. The instrument measured the abundances of galactic cosmic rays in the charge $(Z)$ range $Z \geq 10$ with excellent charge resolution, displaying well resolved individual element peaks for $10 \leq Z \leq 40$. SuperTIGER collected $\sim 3.95 \times 10^{6}$ Iron nuclei, $\sim 7.1$ times as many as detected by TIGER. We will present details of the data analysis techniques and the elemental abundances in the range $30 \leq Z \leq 40$. The data presented contain more than 600 events in this charge range, with charge resolution at ${ }_{26} \mathrm{Fe}$ of $<0.18 \mathrm{cu}$. Our measured abundances are generally consistent with those measured by TIGER and ACE. Our results confirm the earlier results from TIGER, supporting a model of cosmic-ray origin in $\mathrm{OB}$ associations, with preferential acceleration of refractory elements over volatile elements.

This research was supported by NASA under grants NNX09AC17G, NNX14AB25G, the Peggy and Steve Fossett Foundation, and the McDonnell Center for the Space Sciences at Washington University in St. Louis.
\end{abstract}

The 34th International Cosmic Ray Conference,

30 July- 6 August, 2015

The Hague, The Netherlands

\footnotetext{
* Speaker.

${ }^{\dagger}$ We thank the NASA Columbia Scientific Balloon Facility, the NASA Balloon Program Office, and the NSF United States Antarctic Program for the excellent and highly professional efforts that resulted in the record long-duration balloon flight and recovery for SuperTIGER.
} 


\section{Introduction}

The SuperTIGER (Trans-Iron Galactic Element Recorder) instrument is a large-area, balloonborne instrument developed to measure the elemental abundances of Galactic Cosmic Ray (GCR) nuclei from Neon (atomic number $Z=10$ ) to Zirconium $(Z=40)$ with individual-element charge resolution and high statistical precision. SuperTIGER builds on the heritage of the successful TIGER experiment, which flew two Antarctic long-duration balloon missions in 2001 and 2003. SuperTIGER was launched from Williams Field, Antarctica, on December 8, 2012, and flew for 55 days at a mean atmospheric depth of $4.4 \mathrm{~g} / \mathrm{cm}^{2}$. During the flight, SuperTIGER collected over 600 events in the charge range $30<Z \leq 40$ with well-defined single-element peaks.

The SuperTIGER Instrument consists of two nearly-identical modules, each consisting of a suite of seven detectors. Three scintillation detectors measure the differential energy loss, $\frac{d E}{d X}$, within the instrument; two scintillating fiber hodoscopes measure particle trajectory; and two Cherenkov detectors are used to measure Cherenkov emission. From top to bottom, each module consists of a top scintillator detector (S1), the top hodoscope plane (HT or H1), the Aerogel Cherenkov detector $(\mathrm{C} 0)$, the Acrylic Cherenkov detector $(\mathrm{C} 1)$, another scintillator detector (S2), the bottom hodoscope plane (HB or H2), and the bottom scintillator detector (S3). The acrylic Cherenkov (C1) radiators have an index of refraction $n=1.49$, while the Aerogel detectors (C0) have radiation with either $n=1.04$ (for 3 out of 4 half modules of the instrument) or $n=1.025$ (for the remaining half module). These indices correspond to energy thresholds of $300 \mathrm{MeV} /$ nucleon for $\mathrm{C} 1$, and $2.5 \mathrm{GeV} /$ nucleon and $3.3 \mathrm{GeV} /$ nucleon, respectively, for $\mathrm{C}$. Photomultiplier Tubes (PMTs) were used to measure the light output of GCR nuclei within each detector, and the PMT signals were read out with a custom electronics suite. More information on the SuperTIGER instrument can be found in [1]. In these proceedings, more information on the instrument and data analysis can be found in [2] and [3].

\section{Scientific Background}

Previous results from ACE/CRIS [4] and TIGER [5] imply a GCR source consisting of a mix of $\sim 80 \%$ normal interstellar material with Solar System chemical abundances, and $\sim 20 \%$ enriched material from the ejecta from Wolf-Rayet star stellar winds and material created in previous supernovae. These results support the OB association model of GCR origins. TIGER had excellent charge resolution, but had limited statistics at higher charges. TIGER results further emphasized that GCRs originate in the core of superbubbles and that the GCR acceleration process favors elements found in interstellar dust grains (refractory elements) over those found in a gaseous state (volatile elements). This observation fits with the model developed by Meyer, Drury, and Ellison (1997) [6], where these dust grains accumulate a small surface electrical charge, and therefore a very high rigidity (compared with ionized gas), which allows for their efficient acceleration by supernova shocks. Through collisions with atoms in the local interstellar medium, some atoms are sputtered off the grains and injected into the CR accelerator suprathermally. Volatile elements, found in interstellar gasses, do not have the benefit of this suprathermal injection. For these volatile atoms, Ellison, Drury, and Meyer (1997) [7] suggest a rigidity-based acceleration model that is related to the element's mass $(A)$ to ionized charge $(Q)$ ratio. However, no such mass-dependent 
trend is predicted for the refractory elements. When comparing measured GCR source abundances to the mix of Solar System material and massive star outflow, TIGER results clearly show this mass-dependent trend for the volatile elements [5] but also seemed to show a mass-dependent trend among the refractory elements, which was not predicted by Ellison, Drury, and Meyer's model. However, more massive refractory elements such as ${ }_{38} \mathrm{Sr}$ had limited statistics in the TIGER data set. To further test this model, a larger dataset with increased statistics was necessary. The primary scientific goals of the SuperTIGER experiment were to measure the abundances of GCR in the charge range $10 \leq Z \leq 40$ with high statistical accuracy and individual element resolution and to make exploratory measurements of GCR abundances up to ${ }_{56} \mathrm{Ba}$. These measurements will enable us to further test the OB association model of GCR origins and models of GCR acceleration.

\section{Data Analysis Techniques}

Two complementary techniques were used to assign a charge $Z$ to SuperTIGER data events. At low energies (above the $\mathrm{C} 1$ threshold of $\sim 300 \mathrm{MeV} /$ nucleon but below the $\mathrm{C} 0$ threshold of $2.5 \mathrm{GeV} /$ Nucleon or $3.3 \mathrm{GeV} / \mathrm{Nucleon}$, depending on the half-module the event went through), the charge is determined using a combination of signals from the top two layers of scintillator detectors (S1 and S2) and the Acrylic (C1) Cherenkov detector. At energies above the C0 threshold, the charge is determined with a combination of the $\mathrm{C} 1$ and Aerogel $(\mathrm{C} 0)$ detector signals. Before either technique was applied, a mapping correction was applied to the data to remove the effects of position- and angle-dependent differences in detector response. A series of interaction cuts (which required agreement in the signal between various detectors) was also applied to reject events which interacted within the active area of instrument. The SuperTIGER data were divided into two data sets, a high energy (Above $\mathrm{C} 0$ ) set and low energy (Below C0) set, as described above, and each data set was analyzed separately.

\subsection{Below C0}

At low energies, plotting the scintillator signal against the signal from the Acrylic (C1) Cherenkov detector gives clearly defined and separated charge bands, as shown in Figure 1 (left, above). Assigning charge to the Below $\mathrm{C} 0$ data set depends on finding a model of the response of the scintillator light output to higher charges. The SuperTIGER Scintillator Detectors provide a measurement of the amount of light emitted by the radiator as a function of the path length traversed by the ionizing particle, $\frac{d L}{d x}$. The radiator of each scintillator is comprised of a base material, with a small amount of primary and secondary dyes. In an ideal scintillator, the amount of scintillation light produced will be proportional to the energy loss given by the Bethe-Bloch equation, which is proportional to $Z^{2}$ where $Z$ is the charge of the particle. However, when a particle deposits a large amount of energy in a small volume of the scintillator, saturation occurs. This means that the actual energy converted to light as a function of path length $\left(\frac{d L}{d x}\right)$ is a fraction of the stopping power $\left(\frac{d E}{d x}\right)$ that decreases with increasing density of ionization.

The model first proposed by Voltz et al. [8] was found to be the best fit to the SuperTIGER scintillators after numerous models were tried. The Voltz model was also found to be the best fit for the TIGER scintillator detectors and was used in the TIGER analysis. In the Voltz model, the areas of light emission are broken up into a "core" of material near the path of the particle and 


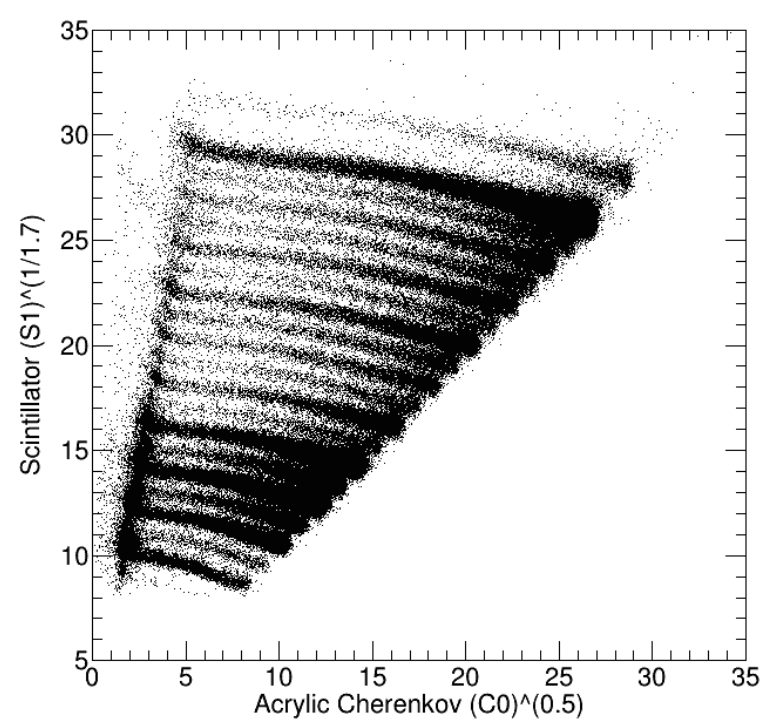

Figure 1: Cross plot comparing Scintillator and Acrylic Cherenkov signals used in lowenergy (Below $\mathrm{C} 0$ ) analysis.

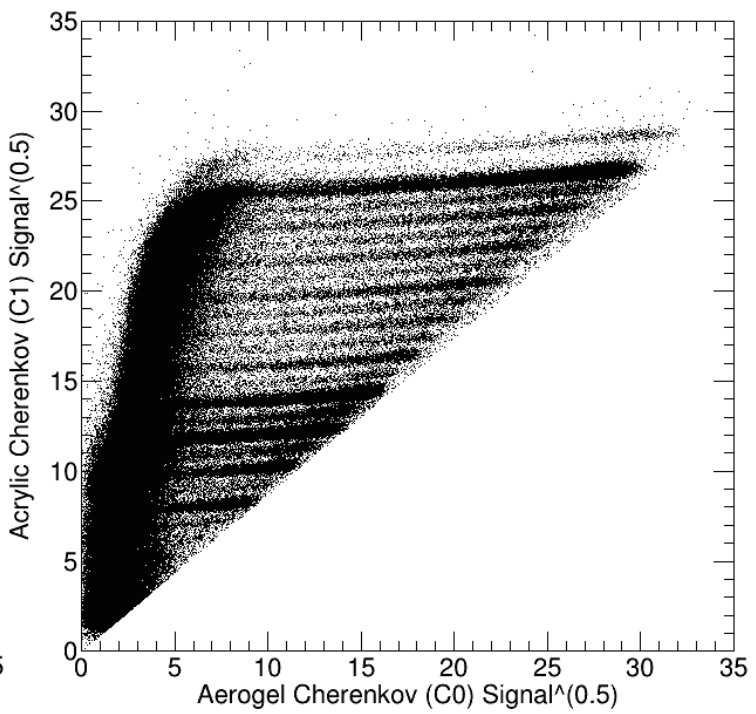

Figure 2: Cross plot comparing Aerogel and Acrylic Cherenkov signals used in highenergy (Above $\mathrm{C} 0$ ) analysis.

a "halo" area further away from the core, which behaves differently. This model is given in the mathematical treatment of Ahlen et al. [9] as:

$$
\frac{d L}{d x}=A_{S} \frac{d E}{d x}\left(1-F_{S}\right) \exp \left[-B_{S}\left(1-F_{S}\right) \frac{d E}{d x}\right]+A_{S} \frac{d E}{d x} F_{S}
$$

Here, $A_{S}$ is an arbitrary scaling parameter, $\frac{d E}{d x}$ is the energy loss calculated from the Bethe-Bloch formula, $B_{S}$ is a parameter describing the quenching behavior, and $F_{S}$ is the fraction of total $\frac{d E}{d x}$ that escapes via knock-on electrons from the core into the halo region. To fit the Voltz model to the SuperTIGER data, we first fit curves of constant charge to each of the well-separated contours shown in Figure 1. We then found curves on the plot of constant $\left(\mathrm{C} 1 / Z^{2}\right) .\left(\mathrm{C} 1 / Z^{2}\right)$ is a function of the energy of the incident particle. In this case, we used the simple approximation that $Z \sim S^{(1 / 1.7)}$, where $S$ is the signal from the scintillator detector. This approximation was sufficient to ensure that each line of constant $\left(\mathrm{C} 1 / Z^{2}\right)$ represented a roughly constant "energy". We then found the intersection of these lines of constant "energy" with the lines of constant charge in order to model the scintillator response as a function of charge $Z$. This process was repeated for both the $\mathrm{S} 1$ and S2 scintillator detectors in each of 30 angle bins.

Using lines of nearly constant "energy" allowed us to use the energy-independent form of Equation 3.1, where $\frac{d E}{d x} \rightarrow C_{B B} Z^{2}\left(C_{B B}\right.$ is a constant from the Bethe-Bloch equation, with $A_{a b}$ and $Z_{a b}$ the Atomic Mass and Number of the target medium) and the equation can be written into a $Z^{2}$ term and a term that describes the energy losses not detected by the detector.

$$
\frac{d L}{d x}=S=A Z^{2}+B Z^{2} e^{-C Z^{2}}
$$




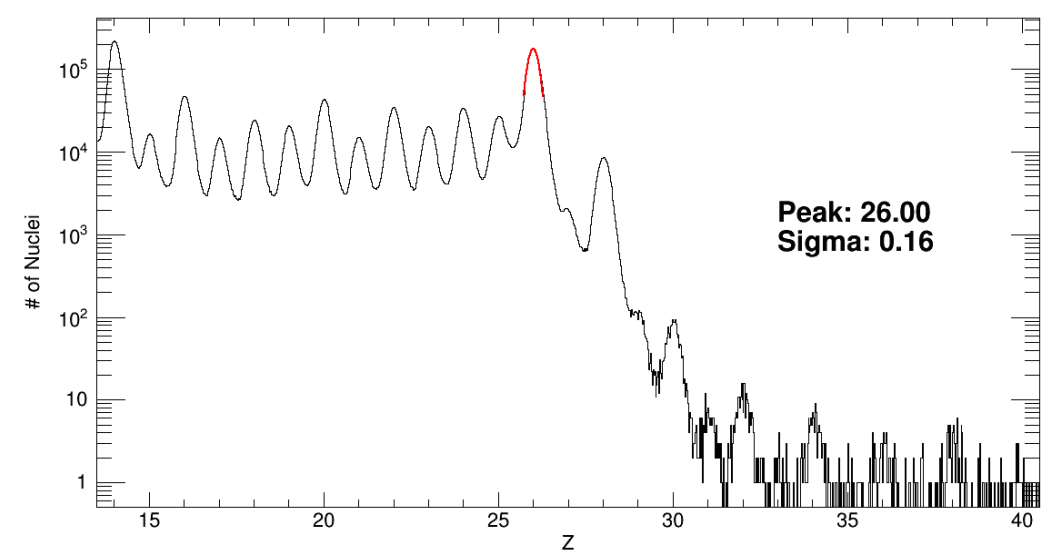

Figure 3: Combined SuperTIGER Charge histogram with 0.025 c.u. bins, showing the peak location and resolution at ${ }_{26} \mathrm{Fe}$.

where:

$$
\begin{gathered}
A=A_{S} C_{B B} F_{S} \quad B=A_{S} C_{B B}\left(1-F_{S}\right) \quad C=B_{S} C_{B B}\left(1-F_{S}\right) \\
C_{B B}=4 \pi N_{A} r_{e}^{2} m_{e} c^{2} \frac{Z_{a b}}{A_{a b} \beta^{2}}\left(\ln \left[\frac{W_{\max }}{I}\right]-\beta^{2}\right)
\end{gathered}
$$

Each line of constant "energy" therefore gave 3 parameters, A, B, and C. We then plotted the value of each parameter as a function of $\left(\mathrm{C} 1 / Z^{2}\right)$ and fit a fourth-order polynomial to model the energy dependence of the parameters. This fit procedure was repeated in each of 30 angle bins. For an event with an arbitrary "energy" (which depends on $\mathrm{C} 1$ and $\mathrm{S}$ signal) and angle, we were then able to assign a unique set of parameters $A, B$ and $C$ to Equation 3.2, and solve for the charge $Z$.

This method was used on both the S1 and S2 scintillators to assign charge. The S3 signal helped identify and reject nuclei that interacted in the instrument, but is not used to measure charge. However, the Voltz model is not a perfect fit to the SuperTIGER scintillator response, and while the method described above gave well-defined, well-separated peaks in a charge histogram, the peaks were not always aligned at integer values of $Z$. Therefore, a slight charge-dependent shift was assigned to both the $S 1$ and $S 2$ charges to ensure that the histogram peaks lined up, and then the final charge $Z$ for the Below $\mathrm{C} 0$ method was calculated as the average of the $\mathrm{S} 1$ and $\mathrm{S} 2$ charges.

\subsection{Above C0}

For events with energy above the C0 threshold (2.5 GeV/Nucleon or $3.3 \mathrm{GeV} / \mathrm{Nucleon}$, depending on the half-module the event went through), the charge is assigned using the Aerogel (C0) and Acrylic (C1) Cherenkov signals. Figure 2 shows that plotting these two signals against each other gives well-defined, well-separated charge bands. The signal from the Cherenkov detectors is given by:

$$
L_{C h}=K Z^{2}\left(1-\frac{1}{\beta^{2} n^{2}}\right)
$$

where $K$ is some constant unique to each detector and $n$ is the index of refraction of the Cherenkov radiator. Assuming a negligible energy loss between these two adjacent detectors $\left(\beta_{C 0}=\beta_{C 1}\right)$, we 


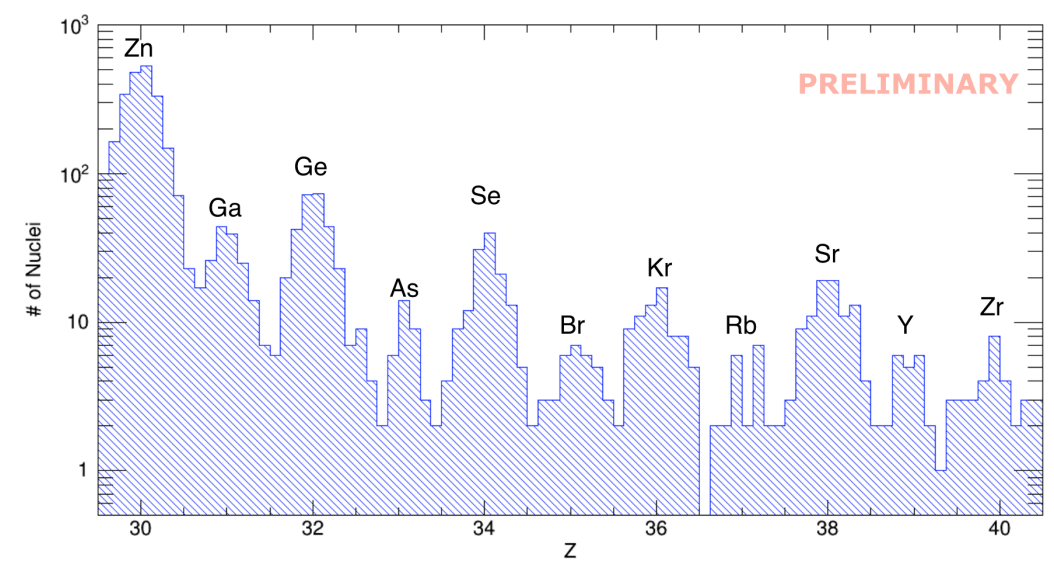

Figure 4: Ultra-Heavy Charge Histogram with 0.125 c.u. bins.

are left with two measured values (the $\mathrm{C} 1$ and $\mathrm{C} 0$ signals) to find two unknowns ( $Z$ and $\beta$ ). In each of 30 angle bins, we isolated the ${ }_{26} \mathrm{Fe}$ charge band on the $\mathrm{C} 1$ vs $\mathrm{C} 0$ cross plot, and then broke this up into 30 bins with different $\mathrm{C} 1$ values with roughly equal numbers of ${ }_{26} \mathrm{Fe}$ events. We then found typical values of $C 0^{0.5} / 26$ and $C 1^{0.5} / 26$ for each bin, plotted these against each other, and fit this plot with a quadratic function. This factored out the $Z^{2}$ dependence of the Cherenkov detector, allowing us to find the $\beta$ dependence. We then applied this $\beta$ correction to the data, and assigned a charge. A further velocity correction was then applied to ensure that the ${ }_{26} \mathrm{Fe}$ peaks in various energy bins were aligned. Similar to the Below $\mathrm{C} 0$ data, we then added a small charge-dependent correction factor to ensure that the well-defined, well-separated peaks this method gave us lined up with integer values of $Z$ in the charge histogram.

\section{Ultra Heavy Abundances}

The charge $Z$ assigned to each event by either the Below- or Above-C0 methods was then placed into a combined histogram, shown in Figures 3 and 4. For events with $Z<33$, this histogram was fit with a multi-peaked gaussian fitting routine initially developed for ACE/CRIS and used for TIGER analysis [10]. For $Z \geq 33$ we simply counted the number of events in each of the welldefined peaks evident in Figure 4. For elements with $Z>29$, we used a less restrictive interaction cut to increase statistics.

After determining the number of nuclei of each element observed within the SuperTIGER instrument, we then corrected for interactions within the instrument using interaction mean free paths for each $Z$ (from [13] and [14]), and the appropriate path length within the instrument for each of the types of material that make up the SuperTIGER detector. We used this simple assumption since our interaction cuts eliminate those events that interact and change charge within the detector. We then determined the Top-of-Instrument abundances, normalized to ${ }_{26} \mathrm{Fe}=1$. The uncertainties assigned to each abundance are statistical. For elements with $N \geq 100$, we simply assigned a $\sqrt{N}$ uncertainty. For elements with $N<100$, we used the upper and lower limits calculated by Gehrels (1986) [15]. 


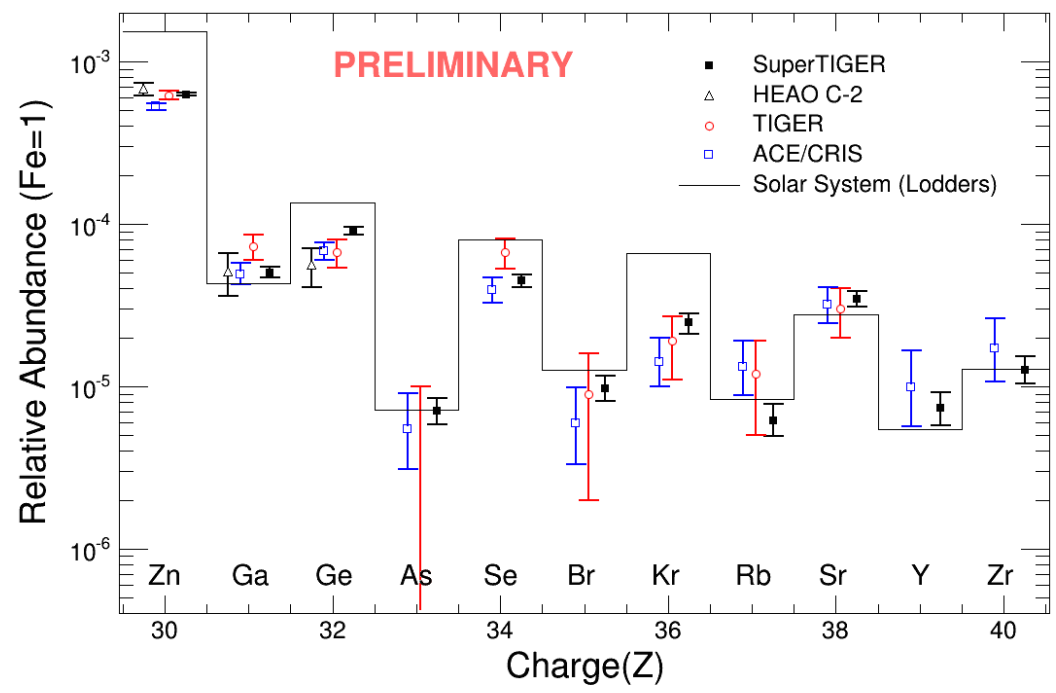

Figure 5: Comparison of SuperTIGER Top-of-Atmosphere abundances with space abundances from ACE/CRIS [11] and HEAO-C3 [12], and top-of-atmosphere abundances from TIGER [5]. SuperTIGER error bars are statistical only.

Using the same atmospheric propagation method and code as used on TIGER, we then calculated the relative abundances of each element at the top of the atmosphere. Figure 5 shows how the SuperTIGER data (again, with purely statistical error bars) compares to Solar System abundances from Lodders [16] and GCR space abundances observed by HEAO-C3, ACE/CRIS, and top-of-atmosphere abundances from TIGER. The SuperTIGER abundances are not inconsistent with previous measurements, and the improved statistics represent a major improvement.

To get a preliminary look at the GCR source abundances, we took these Top-of-Atmosphere abundances and assumed the same GCR source to Top-of-Atmosphere abundance ratio that was calculated for the TIGER data (from Rauch et al.[5]). Figure 6 shows the calculated preliminary GCR source compared to an 80/20 mix of material with Solar System abundances and massive star outflow, along with the TIGER data points. The error bars are still statistical only, so uncertainties in the propagation have not been taken into account. A proper propagation calculation is planned for the near future. The mass-dependent trends in both the volatile and refractory elements remains, with smaller error bars in particular on the refractory ${ }_{38} \mathrm{Sr}$ point. We also plan to include a ${ }_{40} \mathrm{Zr}$ point on the plot in the near future, which will add another high-mass refractory point.

\section{Conclusion}

The SuperTIGER instrument has measured the largest dataset with single-element resolution in the range $30 \leq Z \leq 40$ to date. Preliminary analysis of the over 600 events in the range 30 $<Z \leq 40$ shows that the GCR space and source abundances are consistent with previous observations with significantly increased statistics. Ongoing analysis will provide an important test of the OB association origin of GCR origins and the Volatility model of GCR acceleration. A second SuperTIGER flight is planned and will provide further statistics and tests of GCR origins. 


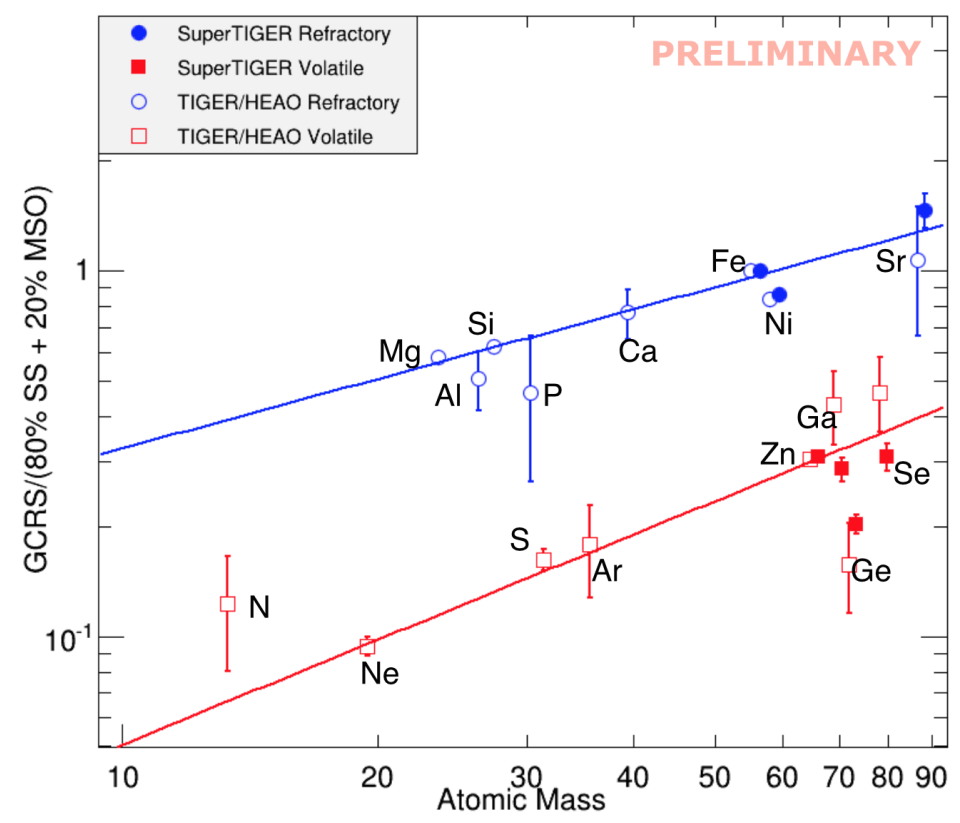

Figure 6: Comparison of SuperTIGER refractory and volatile element GCR source abundances with HEAO and TIGER and predicted GCR source material. SuperTIGER error bars are statistical errors only. SuperTIGER points are slightly offset from TIGER points for clarity. This is an updated version of the plot in [5].

\section{References}

[1] W. R. Binns et al., The SuperTIGER Instrument: Measurements of Elemental Abundances of Ultra-Heavy Galactic Cosmic Rays, ApJ 788 (June 2014) 18.

[2] M. Sasaki et al., Proceedings of the 34th International Cosmic Ray Conference (2015).

[3] A. W. Labrador et al., Proceedings of the 34th International Cosmic Ray Conference (2015).

[4] W. R. Binns et al., Cosmic-Ray Neon, Wolf-Rayet Stars, and the Superbubble Origin of Galactic Cosmic Rays, ApJ 634 (Nov. 2014) 351-364, [astro-ph/0508398].

[5] B. F. Rauch et al., Cosmic Ray origin in OB Associations and Preferential Acceleration of Refractory Elements: Evidence from Abundances of Elements ${ }_{26}$ Fe through ${ }_{34} S e$, ApJ 697 (June 2009) 2083-2088, [arXiv:096.2021].

[6] J. P. Meyer, L. O. Drury, and D. C. Ellison, ApJ, 487 (1997) 182.

[7] D. C. Ellison, L. O. Drury, and J. P. Meyer, ApJ, 487 (1997) 197.

[8] R. Voltz et al., Journal of Chemical Physics, 45 (1966) 3306.

[9] S. P. Ahlen et al., Reviews of Modern Physics 52 (1980) 121-173.

[10] L. M. Scott, Cosmic-Ray Energy Loss in the Heliosphere and Interstellar Reacceleration, Ph.D. Thesis, Washington University in St. Louis (2005).

[11] W.R. Binns et al., Proc. of International Cosmic Ray Conference (Rio de Janerio), (2013) Paper 0646.

[12] B. Byrnak et al., Proceedings of the 18th International Cosmic Ray Conference (1983) 2, 29.

[13] B. S. Nilsen, C. J. Waddington, J. R. Cummings, T. L. Garrard, and J. Klarmann. Phys Rev C. 52, (1995) 3277

[14] G. D. Westfall et al., Fragmentation of Relativistic ${ }^{56} \mathrm{Fe}$, Phys Rev C )19 (1979) 1309-1323

[15] N. Gehrels, Confidence limits for small numbers of events in astrophysical data, ApJ ,303 (1986) 386.

[16] K. Lodders et al., In Landolt-Bornstein, New Series, Vol VI/4B, Chap. 4.4, J. E. Trumper (ed), Springer Verlag (2009) 560. 